\title{
Probing the Functional Activity Limits of Biomolecules Under Electron Irradiation
}

Trevor Moser ${ }^{1}$, James Evans ${ }^{1,2^{*}}$

${ }^{1}$ Environmental Molecular Sciences Laboratory, 3335 Innovation Blvd., Richland, WA USA 99354

${ }^{2}$ School of Biological Sciences, Washington State University, Pullman, WA, 99164, USA

*Corresponding author contact: James.Evans@PNNL.Gov

Liquid cell transmission electron microscopy (LC-TEM) has the potential to allow for high resolution imaging of biological dynamics in a fully hydrated environment. While electrons can allow for subnanometer spatial resolution, one drawback of their use is the unavoidable ionizing radiation damage resulting from inelastic scattering events. In aqueous environments, highly reactive radical species are generated during the damage process which can react with other nearby molecules thereby causing secondary chemical damage in the sample (1). The impact of these radicals on the chemistry of the sample has been a primary point of study for the LC-TEM field, although the effect of radiation driven chemistry changes on the physiology of biological systems is not fully clear. Morphological changes in bacteria irradiated with electrons in a liquid cell has been described $(2,3)$, but the thresholds for impacting biomolecule activity and functionality are not yet known.

While the cryo-EM field has characterized the relationship between electron flux and loss of structure in detail (4), the link between electron irradiation and loss of function is not as clear. Dehydrated catalase monolayers have been demonstrated to lose functional activity at electron fluxes around $0.05 \mathrm{e}^{-} / \AA^{2}(5)$, several orders of magnitude lower then typically used for atomic resolution structure determination for these same samples by cryo-EM. Understanding the critical electron fluxes that result in a significant loss, or alteration, of biological function for proteins, lipids, and DNA in a fully hydrated environment is a necessary step to ensure observed dynamics by LC-TEM are representative of a normal physiological process rather than a beam driven artifact.

This work will demonstrate the functional inactivation thresholds of several biomolecules attached to the top silicon nitride membrane of a liquid cell enclosure as determined by sequentially irradiating them with known electron fluxes. Biomolecule functional activity is then verified optically to quantify functional activity loss relative to increasing electron flux. Figure 1 shows optical images of one such experiment before and after electron irradiation showing the loss of fluorescence activity at a flux well below those typically used in cryo-EM or LC-TEM. We will discuss these results and potential implications for future research. 


\section{References}

1. N. M. Schneider et al., Electron-Water Interactions and Implications for Liquid Cell Electron Microscopy. The Journal of Physical Chemistry C 118, 22373-22382 (2014).

2. T. H. Moser et al., The role of electron irradiation history in liquid cell transmission electron microscopy. Sci Adv 4, eaaq1202 (2018).

3. T. H. Moser, T. Shokuhfar, J. E. Evans, Considerations for imaging thick, low contrast, and beam sensitive samples with liquid cell transmission electron microscopy. Micron 117, 8-15 (2019).

4. L. A. Baker, E. A. Smith, S. A. Bueler, J. L. Rubinstein, The resolution dependence of optimal exposures in liquid nitrogen temperature electron cryomicroscopy of catalase crystals. J Struct Biol 169, 431-437 (2010).

5. M. Hahn, J. Seredynski, W. Baumeister, Inactivation of catalase monolayers by irradiation with $100 \mathrm{keV}$ electrons. Proceedings of the National Academy of Sciences 73, 823-827 (1976).

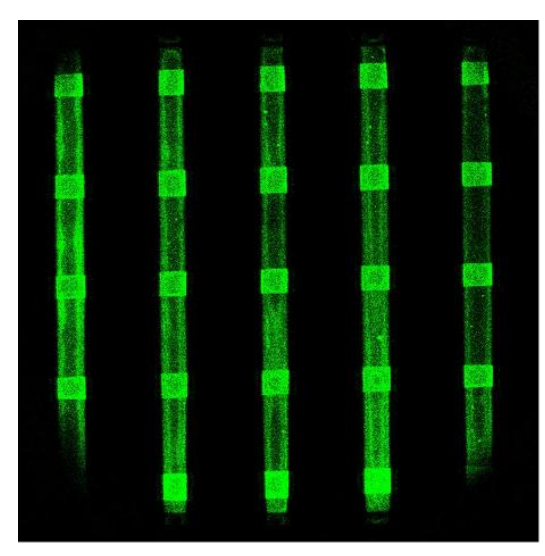

FITC Signal

Pre-Irradiation

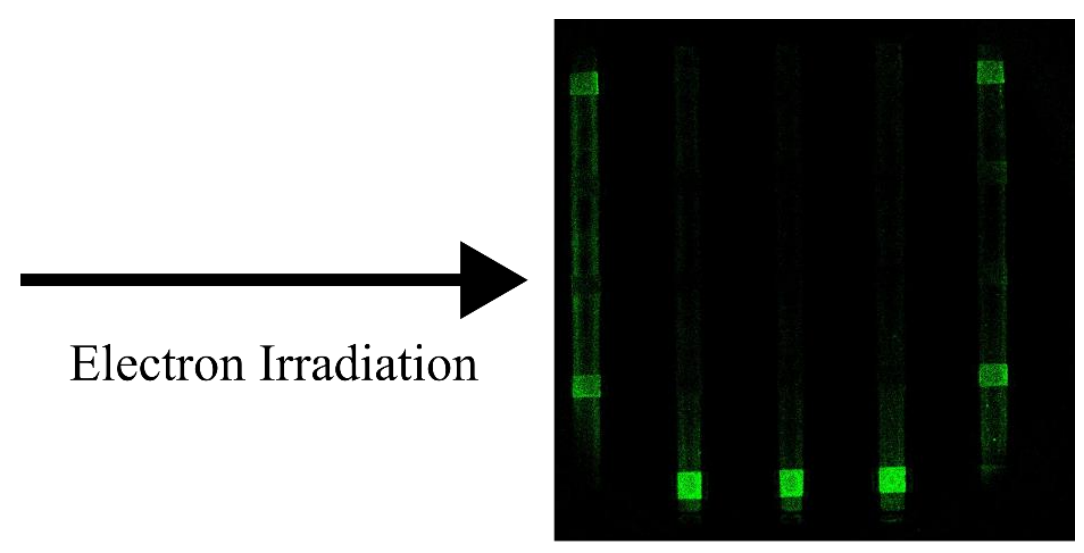

FITC Signal

Post-Irradiation

Figure 1. A lipid monolayer tagged with the fluorescent probe FITC on the silicon nitride membranes of a liquid cell. Optical images show the fluorescence signal of FITC before and after irradiation with the electron beam at a flux of $0.1 \mathrm{e}^{-/} \AA^{2}$. 